\title{
12 POSITIVE AIRWAY PRESSURE ADHERENCE AND REMOTE VERSUS FACE-TO-FACE EDUCATION FOR SLEEP APNOEA DURING THE NOVEL CORONAVIRUS (COVID-19) PANDEMIC
}

'Johan Meurling*, 'Adam Birdseye, ${ }^{1}$ Rohan Gell, ${ }^{1}$ Eliza Sany, ${ }^{1}$ Richard Brown, ${ }^{1}$ Sean Higgins, ${ }^{1}$ Rexford Muza, ${ }^{1,2}$ David O' Regan, ${ }^{1,3,4}$ Guy Leschziner, ${ }^{1,5}$ Joerg Steier, ${ }^{1,3}$ Ivana Rosenzweig, 1,5Panagis Drakatos. 'Sleep Disorders Centre, Guys and St. Thomas' NHS Foundation Trust, London, UK; ${ }^{2}$ School of Medicine, King's College London, London, UK; ${ }^{3}$ Plasticity Centre, Department of Neuroimaging, Institute of Psychiatry, Psychology and Neuroscience (IOPPN), King's College London, London, UK; ${ }^{4}$ Department of Neurology, Guy's and St Thomas' NHS Foundation Trust, London, UK; ${ }^{5}$ Faculty of Life Sciences and Medicine, King's College London, London, UK

\subsection{6/bmjresp-2021-bssconf.10}

Introduction We aim to quantify adherence of positive airway pressure (PAP) initiated during the COVID-19 pandemic and compare the effects of remote versus face-to-face (FTF) education in patients with obstructive sleep apnoea (OSA).

Methods Prospective cohort study in a tertiary National Health Service (NHS) hospital sleep disorders centre in London, United Kingdom, involving 141 patients aged $>18$ years with newly diagnosed OSA initiating PAP during the first national coronavirus (COVID-19) lockdown in 2020. We compare 70 patients educated on PAP remotely to 71 patients receiving standard FTF education. We measured adherence over a continuous 30-day period within the first three months of PAP usage, including average nightly usage, usage per nights used, percentage of nights used, and percentage of nights used for $>4$ hours. 